\title{
Studies of parton thermalization at RHIC
}

\author{
Ghi R. Shin \\ Department of Physics, Andong National University, Andong, South Korea \\ Berndt Müller \\ Department of Physics, Duke University, Durham, NC 27708-0305, USA
}

(Dated: October 29, 2018)

\begin{abstract}
We consider the evolution of a parton system which is formed in the central region just after a relativistic heavy ion collision. The parton consist of mostly gluons, minijets, which are produced by elastic scattering between constituent partons of the colliding nuclei. We assume the system can be described by a semi-classical Boltzmann transport equation, which we solve by means of the test particle Monte-Carlo method including retardation. The partons proliferate via secondary radiative $g g \rightarrow g g g$ processes until the thermalization is reached for some assumptions. The extended system is thermalized at about $t=1.6 \mathrm{fm} / c$ with $T=570 \mathrm{MeV}$ and stays in equilibrium for about $2 \mathrm{fm} / c$ with breaking temperature $T=360 \mathrm{MeV}$ in the rapidity central region.
\end{abstract}




\section{INTRODUCTION}

It is generally expected that a Quark-Gluon Plasma(QGP) can be formed after a relativistic heavy ion collision even at RHIC energy. However, it has not been clear yet how a QGP is formed in a heavy ion collision, as well as what the equation of state is, if it is formed. Some of these questions, which are important for the identification of signals of the QGP and of the properties of dense strongly interacting matter, are the subjects of our study.

Beginning with the moment of contact of two colliding heavy ions, initially coherent quanta, which can be identified as partons, from the projectile nucleus collide with those from the target nucleus and can become incoherent particle excitations. They can, as well, produce additional particles by radiative processes [1, 2]. There may be several different mechanisms responsible for particle production in relativistic heavy ion collision depending on the transverse momentum of the produced particles. Those of high transverse momentum, which is greater than a threshold momentum $p_{0}$, are usually called minijets and can be approximately calculated by the methods of perturbative QCD (pQCD) using the parton structure functions of the colliding nuclei. On the other hand, the "soft" partons of low transverse momentum, with $p_{T} \leq p_{0}$, are thought to be produced by the semi-classical decoherence of strong, random gluon fields contained in the colliding nuclei, sometimes called color glass condensate [3, 4, 5$]$.

While the primary partons of high transverse momentum (minijets) are produced near the central rapidity region and have relatively high transverse energy, the soft partons are distributed over a wide range of rapidities and have lower transverse energy. These soft partons can play a role as a thermal bath or background field, which slows down the minijets by inducing them to transfer a substantial fraction of their energy to the bath, thus enhancing their chance to be thermalized. It was pointed out [6, 7], however, that the major mechanism of energy deposition is through radiative $(2 \rightarrow 3)$ processes, suggesting that it is sufficient to consider a minijet system in order to explore the chemical and thermal equilibration of the system. This is what we are doing here.

We study the initial phase-space distribution of minijets assuming no correlation between momentum and space and another one with correlation, especially those given by Cooper at al[10], which we will call CMN distribution, in Section II. We briefly describe the 
semi-classical Boltzmann equations of motion and the Monte-Carlo simulations to solve the equations in Section III. We present the results of our numerical solutions and a summary in Section IV.

\section{INITIAL DISTRIBUTIONS}

The hard gluons produced after a relativistic heavy ion collision have been studied extensively by many authors. Its momentum distribution as well as the total number of partons can be calculated by the method of pQCD. To see the dominant effect at very early times after the onset of a relativistic heavy ion collision, we consider only minijet gluons. Using the parton distribution of colliding nucleus $A, f_{i / A}\left(x, Q^{2}\right)=f_{i / N}\left(x, Q^{2}\right) R_{A}\left(x, Q^{2}\right)$, where $f_{i / N}\left(x, Q^{2}\right)$ is the parton distribution of a free nucleon and $R_{A}\left(x, Q^{2}\right)$ the nuclear ratio function, the minijet distribution can be calculated by the $2 \rightarrow 2$ minijet cross section per nucleon in a $A+A$ collision,

$$
\frac{d N^{j e t}}{d p_{T} d y}=K T(b) \int d y_{2} \frac{2 \pi p_{T}}{\hat{s}} \sum_{i j, k l} x_{1} f_{i / A}\left(x_{1}, p_{T}^{2}\right) x_{2} f_{j / A}\left(x_{2}, p_{T}^{2}\right) \sigma_{i j \rightarrow k l}(\hat{s}, \hat{t}, \hat{u}),
$$

where $K$ denotes a factor to include the higher order diagrams, which we will set to $K=2$ throughout our study. $T(b)$ is the nuclei geometric factor at an impact parameter $b$. We use the GRV98 set of parton distributions for a free nucleon [11] and the EKS98 parametrization for the ratio function [12]. $x_{1}$ and $x_{2}$ are the Bjorken scaling variables of parton $i$ and $j$ in nucleons of two colliding nuclei, respectively. $y_{1}$ and $y_{2}$ are the rapidities of scattered or produced partons.

The $g g$ scattering cross section at leading order is

$$
\sigma_{g g \rightarrow g g}=\frac{9 \pi \alpha_{s}^{2}}{2 \hat{s}}\left[3-\frac{\hat{t} \hat{u}}{\hat{s}^{2}}-\frac{\hat{u} \hat{s}}{\hat{t}^{2}}-\frac{\hat{s} \hat{t}}{\hat{u}^{2}}\right]
$$

where the "hat" symbol on the Mandelstam variables indicates those of a parton. The relations between the variables are given by

$$
\begin{aligned}
x_{1} & =p_{T}\left(e^{y_{1}}+e^{y_{2}}\right) / \sqrt{s}, \\
x_{2} & =p_{T}\left(e^{-y_{1}}+e^{-y_{2}}\right) / \sqrt{s}, \\
\hat{s} & =x_{1} x_{2} s \\
\hat{t} & =-p_{T}^{2}\left(1+e^{y_{2}-y_{1}}\right), \\
\hat{u} & =-p_{T}^{2}\left(1+e^{y_{1}-y_{2}}\right) .
\end{aligned}
$$


We also rewrite the available kinematic region for convenience [13],

$$
\begin{array}{r}
p_{0}{ }^{2} \leq p_{T}{ }^{2} \leq\left(\frac{\sqrt{s}}{2 \cosh y}\right)^{2}, \\
-\log \left(\frac{\sqrt{s}}{p_{T}}-e^{-y}\right) \leq y_{2} \leq \log \left(\frac{\sqrt{s}}{p_{T}}-e^{-y}\right), \\
|y| \leq \log \left(\frac{\sqrt{s}}{2 p_{0}}+\sqrt{\left.\frac{s}{4 p_{0}^{2}}-1\right)} .\right.
\end{array}
$$

We consider only a head-on collision $(b=0)$ and use the leading-order expression for the running coupling constant $\alpha_{s}=4 \pi\left[b_{0} \log \left(Q / \Lambda_{\mathrm{QCD}}\right)\right]^{-1}$, where $Q$ is the momentum transfer, $b_{0}=11-\frac{2}{3} n_{f}$, and $\Lambda_{\mathrm{QCD}}=200 \mathrm{MeV}$ and $n_{f}=3$. The production of minijet gluons strongly depends on the transverse momentum cut $p_{0}$. We use $p_{0}=1 \mathrm{GeV}$ at RHIC energy, which is marginal for the use of pQCD. Note that we set the minimum transverse momentum to be equal to the saturation momentum, where the gluon distribution becomes semi-classical 5]. The total numbers of minijets (integrated over rapidity) is about 4800 at RHIC. These numbers are quite large since we set the momentum cutoff for the minijets at the lower limit of the range $p_{0}^{2} \sim 1-2 \mathrm{GeV}^{2}$ at RHIC. The rapidity distribution is shown in Fig. 1 of reference [10].

To obtain the phase space distribution of minijets, we first assume that there are no correlation between the momentum and space coordinates of a produced parton. The momentum distribution can be given by the minijet distribution, Eq. (2.1),

$$
f\left(p_{T}, y\right)=C \frac{1}{p_{T}^{2}} \frac{d N^{j e t}}{d y d p_{T}} .
$$

We can choose $\left(p_{T}, y\right)$ using this distribution and the azimuthal angle $\phi$ with equal weight between $(0,2 \pi)$ by the Monte-Carlo method, by which we select the energy and momentum of the produced parton. The spatial distribution of minijets can be deduced from the fact that the minijets are produced from elastic scattering between constitutient partons of two colliding nuclei. Assuming the space-time point of produced particle is just that of elastic scattering, we can estimate the space-time positions based on the classical picture as follows. We assume that the probability for having an elastic scattering of constituent partons is proportional to the nuclear density overlap of the colliding nuclei. The transverse position of elastic scatterings is thus obtained by

$$
\begin{aligned}
& x=r \sin \theta \cos \phi, \\
& y=r \sin \theta \sin \phi,
\end{aligned}
$$


where the distribution of constant nuclear density in the nuclear rest frame is $P(r, \theta, \phi)=$ $3 r^{2} /\left(4 \pi R^{3}\right)$.

To obtain the longitudinal position and the time of a collision, we consider a sphere of radius $R$ which has a constant density and is moving with a constant velocity $v \approx c$. We assume two identical spheres suffering a head-on collision in the CM frame. At a given transverse collision position $(x, y)$, we consider longitudinal tubes of area $\Delta A$ through the point $(x, y)$. Because of the Lorentz contraction, the half-length of the tube is $D / \gamma$ with $D=\sqrt{R^{2}-x^{2}-y^{2}}$ and $\gamma=\left(1-v^{2} / c^{2}\right)^{-1 / 2}$. The tubes from both nuclei begin to overlap one another starting at $t_{s}=(R-D) / \gamma v$ and ending at $t_{e}=(R+D) / \gamma v$. Note that we set the time of first contact between the two colliding nuclei at $t=0$. At time $t$, the overlap volume is given by

$$
\Delta V=2 \Delta A v\left(t-t_{s}\right) / \gamma .
$$

The maximun overlap volume is $\Delta V=2 \Delta A D / \gamma$ at $t=R / \gamma v$. The probability density which elastic parton collisions occur is proportional to the overlap volume and the product of the two Lorentz contracted parton densities:

$$
P(t)=\frac{\gamma^{2} v^{2}}{2 D^{2}}\left(t-t_{s}\right)
$$

Using this collision time distribution, we select the collision time randomly:

$$
t=t_{s}+\frac{2 D}{\gamma v} \sqrt{a_{1}}
$$

where $a_{1} \in[0,1]$ is a random number. Once we have $t$, we can calculate the overlap volume, Eq.(2.14), and its longitudinal range, $-v\left(t-t_{s}\right)<z<+v\left(t-t_{s}\right)$. We again choose the longitudinal location $z$ of the collision in this range randomly:

$$
z=v\left[2\left(t-t_{s}\right) a_{2}-\left(t-t_{s}\right)\right]
$$

where $a_{2} \in[0,1]$ is another random number.

We also consider the phase-space distributions (2.1) at RHIC which were recently proposed by Cooper, Mottola and Nayak[10] (CMN Distribution). The boost noninvariant distribution, for example, is given

$$
f\left(p_{T}, \eta, y, \tau_{0}\right)=C \frac{d N^{\mathrm{jet}}}{d y d p_{T}} \frac{e^{-(y-\eta)^{2} / \sigma^{2}\left(p_{T}\right)}}{\tau_{0} p_{T}^{2} \sigma\left(p_{T}\right) e^{\sigma^{2}\left(p_{T}\right) / 4}},
$$




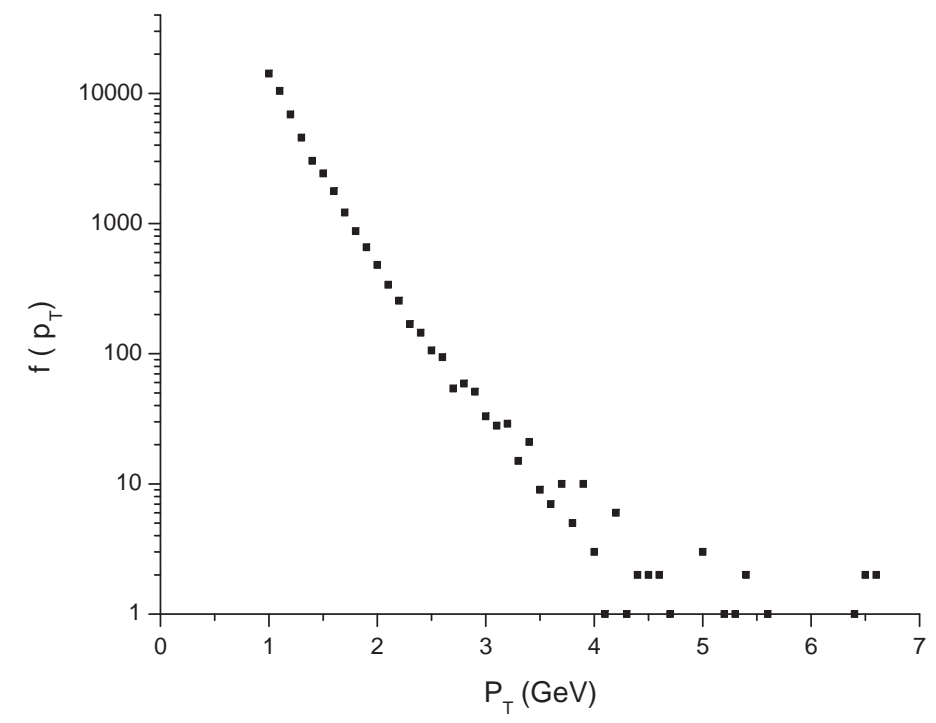

FIG. 1: The transverse momentum distribution of a Monte Carlo sampled parton system as a function of transverse momentum in $\mathrm{GeV}$ unit.

where $\eta$ is spatial rapidity. $\sigma\left(p_{T}\right)$ is an unknown parameter function, which relates the spacerapidity correlation to the transverse momentum and was chosen to be constant $\left(\sigma^{2}=0.28\right)$ at RHIC energy in Ref. [10]. Using this distribution function, we can randomly selects the phase-space coordinates $\left(p_{T}, y, \eta\right)$ of each produced parton. We assume in this distribution that the parton production time is given by Eq. (2.16).

Figure 1 shows the transverse momentum distribution of Monte-Carlo sampled partons with a minimal transverse momentum $p_{0}=1 \mathrm{GeV} / c$. Most of the produced particles have the transverse monenta less than $3 \mathrm{GeV} / c$ and the number of produced gluons sharply increases as the transverse momentum gets smaller.

Figure 2 shows the rapidity distribution of Monte-Carlo sampled particles, which can be compared to the rapidity distribution given in Ref. 10]. The distribution shows that the rapidity is flat in the central region, but falls off for $|y|>3$.

Since we use Monte-Carlo sampling of the distribution, we use several different realizations of the initial distribution (events) and average over the events to get the physical values. 


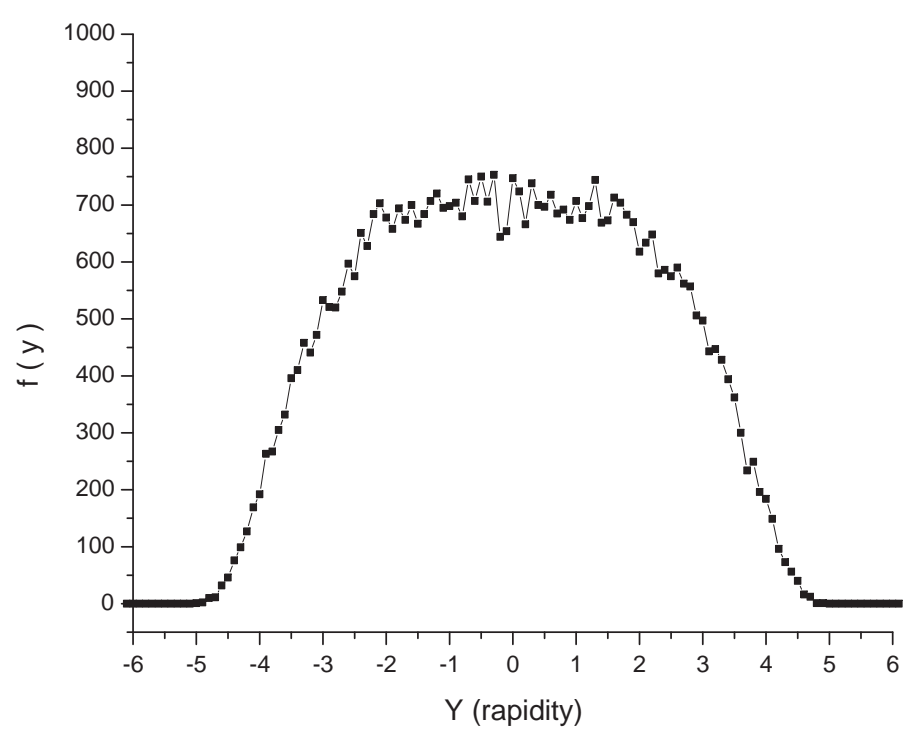

FIG. 2: The rapidity distribution of the produced partons.

\section{NUMERICAL SOLUTION}

The initial-state gluons, which we will call primary gluons from now on, evolve according to the Boltzmann equation [9, 14, 15, 16, 17, 18],

$$
\begin{aligned}
p^{\mu} \partial_{\mu} f_{g}(x, \vec{p}) & =\int_{2} \int_{3} \int_{4} \frac{1}{2} W_{g g \rightarrow g g}\left[f_{g}(3) f_{g}(4)-f_{g}(1) f_{g}(2)\right] \\
& +\int_{2} \int_{3} \int_{4} W_{g q \rightarrow g q}\left[f_{g}(3) f_{q}(4)-f_{g}(1) f_{q}(2)\right] \\
& +\int_{2} \int_{3} \int_{4} \int_{5} \frac{1}{6} W_{g g \rightarrow g g g}\left[f_{g}(4) f_{g}(5)-f_{g}(1) f_{g}(2)\right] \\
& +\int_{2} \int_{3} \int_{4} W_{g \bar{q} \rightarrow g \bar{q}}\left[f_{g}(3) f_{\bar{q}}(4)-f_{g}(1) f_{\bar{q}}(2)\right] \\
& +\int_{2} \int_{3} \int_{4} \frac{1}{2}\left[W_{q \bar{q} \rightarrow g g} f_{q}(3) f_{\bar{q}}(4)-W_{g g \rightarrow q \bar{q}} f_{g}(1) f_{g}(2)\right],
\end{aligned}
$$

where we used the abbreviated notations $f_{g}(i)=f_{g}\left(\vec{x}, \vec{p}_{i} ; t\right), q=(u, d, s), \bar{q}=(\bar{u}, \bar{d}, \bar{s})$, and $\int_{i} \equiv \int d \vec{p}_{i} / E_{i}$. The transition rates can be expressed as

$$
W_{g g_{1} \rightarrow g^{\prime} g_{1}^{\prime}}=s \sigma(s, \Theta) \delta^{(4)}\left(p+p_{1}-p^{\prime}-p_{1}^{\prime}\right)
$$

Note that we assign the label 1 to the momentum $\vec{p}$. We explicitly include the particle symmetry factor in the classical limit. The $g g g \rightarrow g g$ process is not included in the equation 
of motion since the system of high energy partons expands very rapidly so that the available volume increases and the channel is suppressed strongly.

On the other hand, the secondary quarks and antiquarks produced in parton collisions satisfy the equations of motion,

$$
\begin{aligned}
p^{\mu} \partial_{\mu} f_{q}(x, \vec{p}) & =\int_{2} \int_{3} \int_{4} C W_{q q^{\prime} \rightarrow q q^{\prime}}\left[f_{q}(3) f_{q^{\prime}}(4)-f_{q}(1) f_{q^{\prime}}(2)\right] \\
& +\int_{2} \int_{3} \int_{4} W_{g q \rightarrow g q}\left[f_{q}(3) f_{g}(4)-f_{q}(1) f_{g}(2)\right] \\
& +\int_{2} \int_{3} \int_{4} \frac{1}{2}\left[W_{g g \rightarrow q \bar{q}} f_{g}(3) f_{g}(4)-W_{q \bar{q} \rightarrow g g} f_{q}(1) f_{\bar{q}}(2)\right] . \\
p^{\mu} \partial_{\mu} f_{\bar{q}}(x, \vec{p}) & =\int_{2} \int_{3} \int_{4} C W_{\bar{q} \bar{q}^{\prime} \rightarrow \bar{q} \bar{q}^{\prime}}\left[f_{\bar{q}}(3) f_{\bar{q}^{\prime}}(4)-f_{\bar{q}}(1) f_{\bar{q}^{\prime}}(2)\right] \\
& +\int_{2} \int_{3} \int_{4} W_{g \bar{q} \rightarrow g \bar{q}}\left[f_{\bar{q}}(3) f_{g}(4)-f_{\bar{q}}(1) f_{g}(2)\right] \\
& +\int_{2} \int_{3} \int_{4} \frac{1}{2}\left[W_{g g \rightarrow q \bar{q}} f_{g}(3) f_{g}(4)-W_{q \bar{q} \rightarrow g g} f_{\bar{q}}(1) f_{q}(2)\right] .
\end{aligned}
$$

where $C=1 / 2$, if the final state consists of identical particles and $C=1$ otherwise. We neglect the quantum mechanical effects from Bose enhancement factors $\left(1+f_{g}\right)$ for a gluon in the final state and Pauli blocking factors $\left(1-f_{q}\right)$ for final-state quarks or antiquarks.

We use the parton cascade code, which was developed by us and described in ref. [9], to solve the equations of motion for a given initial state. We first initialize all primary partons in space-time with their four-momenta and look for the next possible collision. If a collision occurs, we select the collision products and their new momenta according to the total and differential cross section, respectively, at the space-time of relativistic maximum force point between the colliding particles. This procedure is terminated when the evolution time of the cascade is greater than the final time set as an input parameter.

\section{RESULTS AND CONCLUSION}

Fig. 3 shows the ratio $\sum\left|p_{x}\right| / \sum\left|p_{z}\right|$ in a small sphere with radius $R=1.1 \mathrm{fm}$ at the center of the coordinate frame as a function of time, where

$$
\sum\left|p_{x}\right|=\int d^{3} x \int d^{3} p \frac{\left|p_{x}\right|}{E} f(x, p) .
$$


When this ratio becomes unity, an isotropic, equilibrated configuration has been reached.

As expected, the ratio for the evolution without collisions remains clearly different from unity, which means that there is no kinetic equilibration. The ratio with collisions shows the kinetic isotropy from a time of about $8 \mathrm{GeV}^{-1}=1.6 \mathrm{fm} / c$ to $17 \mathrm{GeV}^{-1}=3.4 \mathrm{fm} / c$ for the system initialized with minijet partons. The simulation includes small angle elastic scattering, for which we set the minimum momentum transfer to $p_{\text {cut }}=0.5 \mathrm{GeV} / c$. We also show the results of simulations with hard scattering only, $p_{\text {cut }}=1 \mathrm{GeV} / c$. Even though the total number of partons in the system is almost same with 9700 , only the system with small angle scatterings reaches a kinetically isotropic state.

On the other hand, the ratio for the system of minijets of CMN distribution always remains away from unity. We can understand this result as follows: Since the value of the spatial rapidity of a produced parton is close to its momentum rapidity, those partons which have positive longitudinal momentum, will be produced with positive longitudinal position, the right hand side of the collision plane, and the parton with negative longitudinal momentum will be born at a negative longitudinal position, on the left-hand side of the collision plane. This correlation thus makes possible only those collisions between the particles which are running in the same longitudinal direction but different transverse momentum. This phenomena reduces the number of collisions and the collision energy substantially, so that the evolution is essentially free streaming.

Figure 4 shows the number density in the sphere as function of time. The density of partons for minijets simulations decreases faster than $1 / t$ and from $32 \mathrm{fm}^{-3}$ at $t=8 \mathrm{GeV}^{-1}$ to $11 \mathrm{fm}^{-3}$, which is much higher than the density of valence quarks of a free nucleon, at $t=17 \mathrm{GeV}^{-1}$ in the small sphere at the center. The density equals to the valence quark density of a free nucleon at $t=34 \mathrm{GeV}^{-1}$. Note that we plot the data if the particle density of the sphere is greater than that of valence quarks in a nucleon, $1.4 \mathrm{fm}^{-3}$.

Figure 5 shows the energy density of the sphere as a function of time, which is almost exponential decreasement. The energy density decreases from $54 \mathrm{GeV} / \mathrm{fm}^{3}$ at $t=8 \mathrm{GeV}^{-1}$ to $12 \mathrm{GeV} / \mathrm{fm}^{3}$ at $t=17 \mathrm{GeV}^{-1}$ for our minijet simulations.

Figure 6 shows the energy per parton in the sphere as a function of time. The energy per parton of free streaming(no collision) is much higher at early stage of expansion than those of interacting systems, which means the parton loses a substantial amount of energy via scattering. The energy decreases from $1.7 \mathrm{GeV}$ at $t=8 \mathrm{GeV}^{-1}$ to $1.1 \mathrm{GeV}$ at $t=17$ 


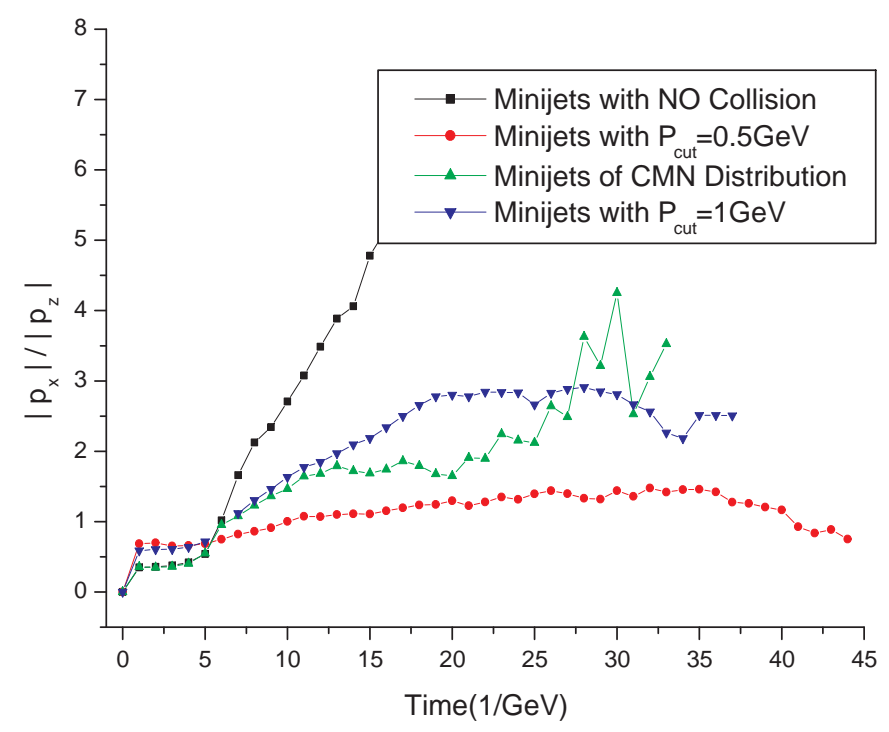

FIG. 3: The ratio $\frac{\sum\left|p_{x}\right|}{\sum \mid p_{z}}$ in a small test sphere $\left(r=1.1 \mathrm{fm}^{3}\right)$ at center region.

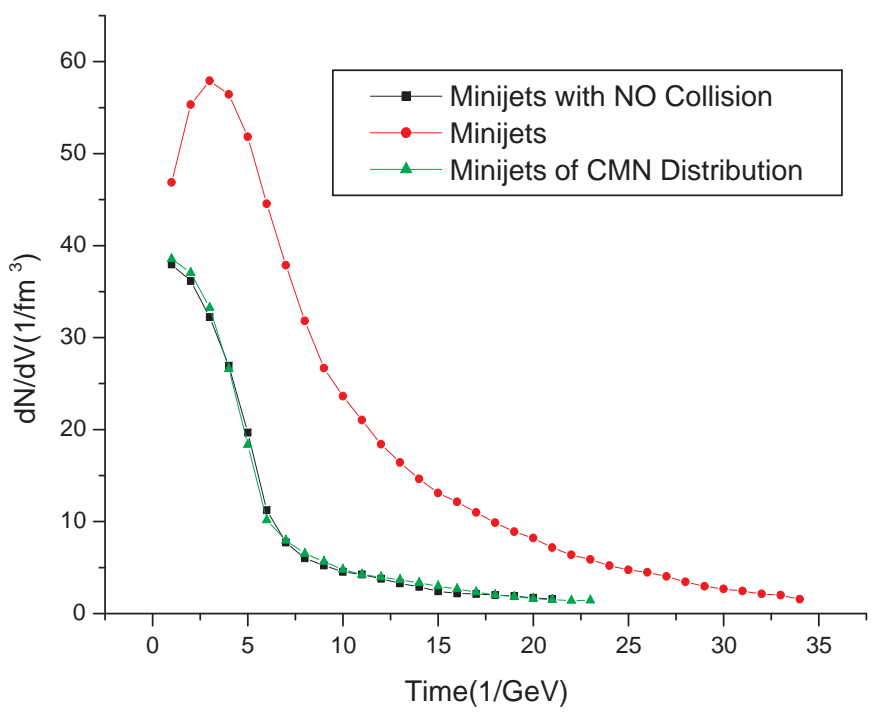

FIG. 4: The particle density $N / V$ in a small test sphere at central region.

$\mathrm{GeV}^{-1}$ for a minijet system. If we assume from Fig 3 that the local system of the sphere at the center is thermalized, the relativistic gas has the temperature $T=566 \mathrm{MeV}$ at $t=8$ $\mathrm{GeV}^{-1}$ to $367 \mathrm{MeV}$ at $t=17 \mathrm{GeV}^{-1}$ for a minijet system and a mixed system, respectively. This system is much hotter than the QGP transition temperature $T_{c} \approx 170 \mathrm{MeV}$. 


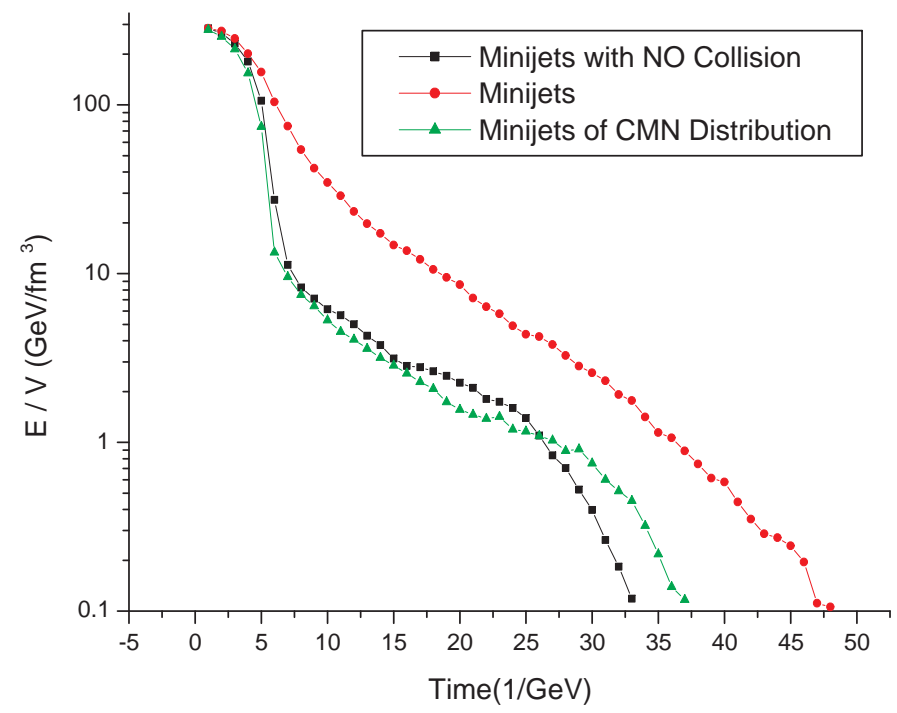

FIG. 5: The energy density $E / V$ of the sphere.

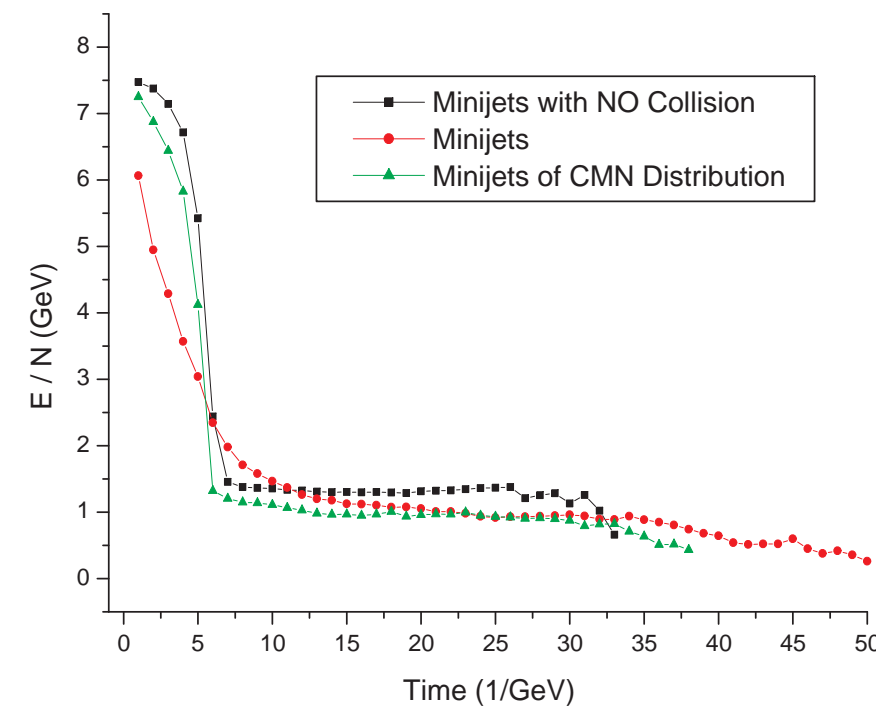

FIG. 6: The energy per particle $E / N$ in the sphere.

Figure 7 shows the total number of particles in the system. The secondary particles are produced within $2 \mathrm{fm} / c$ after the first contact of two colliding nuclei. This shows that the chemical freeze-out comes much earlier than the thermal freeze-out even at the center of collision. The total number of particles is about 9700 for a minijet system and 5200 for 


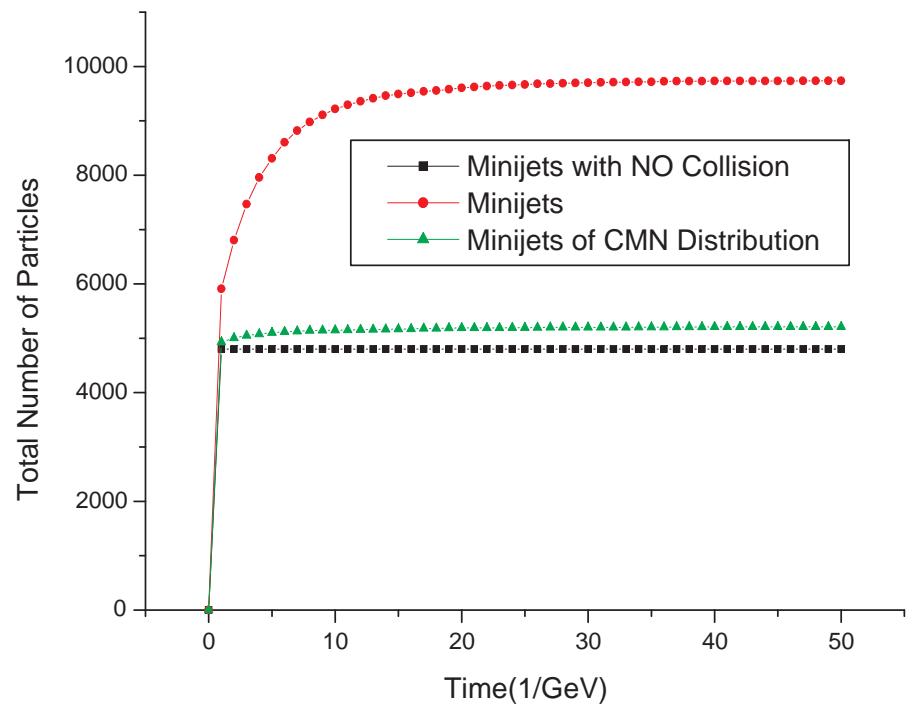

FIG. 7: The total number of particles in the system as function of time.

a minijet system of CMN distribution. As many authors have pointed out, the $g g \rightarrow g g g$ process, even though the process has the kinematical constraint, $\theta\left(p_{T} \lambda_{f}-\cosh y\right) \theta(\sqrt{s} / 2-$ $\left.p_{T} \cosh y\right)$, which is $E_{\mathrm{cm}}>1.54 \mathrm{GeV}$ in our simulations, plays a dominant role in depositing energy and thermalization of a system.

Fig. 8 shows the pseudo rapidity distribution of all of partons in the system at $t=50$ $\mathrm{GeV}^{-1}$. Since partonic collisions are ceased by this time, the distribution will be preserved to the final state unless the hadronic interaction changes them. We can see that the rapidity distribution is narrowed since the high rapidity partons at early time, Fig. 2, have been scattered to produce the low rapidity partons. We also point out that the rapidity distribution is different from the final state (hadronic) rapidity distribution in number and the width[19].

We conclude that the system is thermalized even with minijets at RHIC energy if the small angle scatterings are included. When we look at the small sphere of radius $R=1.1 \mathrm{fm}$ at center, the system is thermalized from $t=1.6 \mathrm{fm} / c$ till about $3.4 \mathrm{fm} / c$. And the temperature is decreasing from 566 to $367 \mathrm{MeV}$ and the particle density from 32 to $11 \mathrm{fm}^{-3}$. Even after this time, the system is still hot untill about $8 \mathrm{fm} / c$ at the center and may cool down further after hadronization. The evolution of the system, which has no correlation between position and momentum, is very similar in character to the "bottom-up thermalization" scenario of 


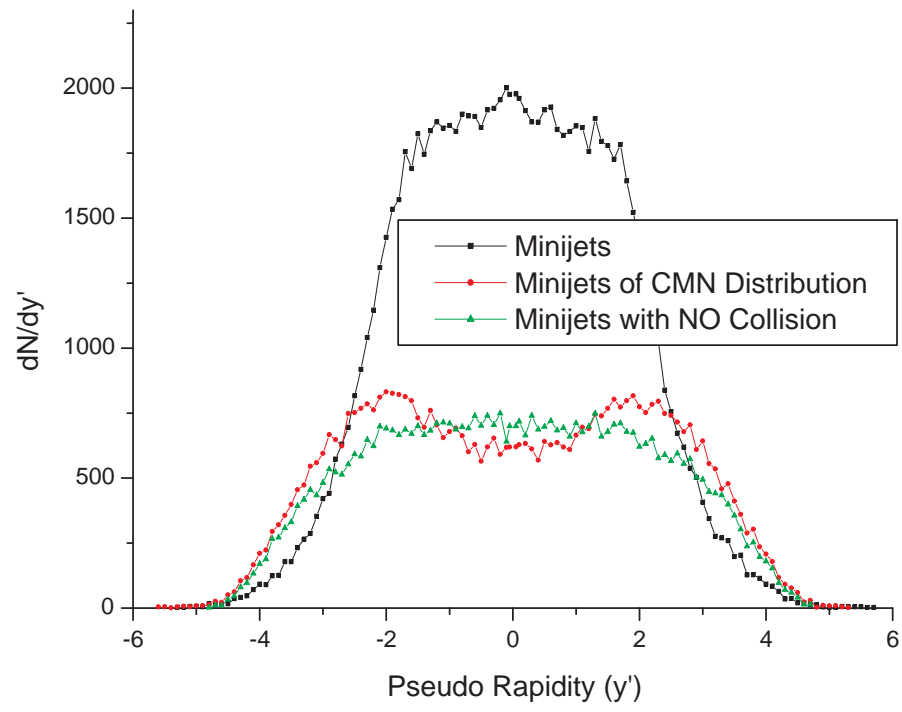

FIG. 8: The pseudo rapidity distribution of parton systems as functions of time.

Baier et al. [7], in which the hard gluons liberated from the colliding nuclei within $\tau \approx Q_{s}^{-1}$ collide among themselves to produce soft gluons and thermalize via small angle scattering.

*Acknowledgements: G. R. Shin was supported in part by grant No. 2000-1-111000-007-1 from the Basic Research Program of the Korea Science and Engineering Agency.

[1] J. D. Bjorken, Phys. Rev. D 27, 140 (1983).

[2] J. P. Blaizot and A. Mueller, Nucl. Phys. B289, 847 (1987).

[3] L. McLerran and R. Venugopalan, Phys. Rev. D 49, 2233 (1994); 49, 3352 (1994); 50, 2225 (1994)

[4] A. Mueller, Nucl. Phys. B572, 227(2000).

[5] A. Krasnitz and R. Venugopalan, Phys. Rev. Lett. 84, 4309(2000); A. Krasnitz and R. Venugopalan, Phys. Rev. Lett. 86, 1717(2001); A. Krasnitz, Y. Nara and R. Venugopalan, PRL 87, 192302(2001); hep-ph/0209269

[6] T. S. Biro, E. van Doorn, B. Müller, M. H. Thoma and X. N. Wang, Phys. Rev. C 48, 1275 (1993).

[7] R. Baier, A. H. Mueller, D. Schiff and D. T. Son, hep-ph/0009237. 
[8] D. Molnar and M. Gyulassy, PRC62(2000), 054907.

[9] G. R. Shin and B. Mueller, J. Phys. G 28, 2643 (2002).

[10] F. Cooper, E. Mottola and G. Nayak, hep-ph/0210391.

[11] M. Glueck, E. Reya, A. Vogt, hep-ph/9806404.

[12] J. Eskola, V.J. Kolhinen and C.A. Salgado, Eur. Phys. J. C9 (1999) 61, hep-ph/9807297, K.J. Eskola, V.J. Kolhinen and P.V. Ruuskanen, Nucl. Phys. B535 (1998) 351, hep-ph/9802350.

[13] N. Hammon, H. Stocker, W. Greiner, hep-ph/9903527, G. C. Nayak, A. Dumitru, L. McLerran, W. Greiner, NP A687(2001) 457.

[14] S.R. de Groot, W.A. van Leeuwen and Ch.G. van Weert, Relativistic Kinetic Theory (NorthHolland, Amsterdam, 1980).

[15] L. P. Csernai, Introduction to Relativistic Heavy Ion Collisions, John Wiley \& Sons, 1994.

[16] K. Geiger and B. Müller, Nucl. Phys. B 369, 600 (1992); K. Geiger, Phys. Rev. D 46, 4965, and 4986 (1992); K. Geiger and J. I. Kapusta, Phys. Rev. D 47, 4905 (1993).

[17] K. Geiger, Phys. Rept. 258, 237 (1995).

[18] B. Zhang, Comp. Phys. Comm. 109, 70 (1997); B. Zhang and Y. Pang, Phys. Rev. C 56, 2185 (1997); B. Zhang, M. Gyulassy and Y. Pang, Phys. Rev. C 58, 1175 (1998).

[19] P. Jacobs, hep-ex/0211031. 